\title{
Lorenzen between Gentzen and Schütte
}

\author{
Reinhard Kahle and Isabel Oitavem
}

\begin{abstract}
We discuss Lorenzen's consistency proof for ramified type theory without reducibility, published in 1951, in its historical context and highlight Lorenzen's contribution to the development of modern proof theory, notably by the introduction of the $\omega$-rule.
\end{abstract}

"Ihr Vorschlag, die Beweismittel nicht ,finit', sondern ,konstruktiv" zu nennen, hat wie eine Art Erlösung auf mich gewirkt."

Paul Lorenzen to Paul Bernays, 1947

\section{Introduction: Hilbert's Programme after Gentzen}

David Hilbert had proposed, in the 1920s, his well-known programme to develop finitist consistency proofs for formalized mathematical theories. Gödel (1931) showed that this programme cannot be carried out in its original form. However, by liberalizing the finitist base of Hilbert's Programme, Gentzen (1936) was able to give a consistency proof for formalized number theory. It satisfies, to a good extent, Hilbert's original aims, when Gentzen uses transfinite induction up to the ordinal $\varepsilon_{0}$ in a controlled way. It is, in particular, justified from a constructive, rather than a finitist, point of view. ${ }^{1}$

Reinhard Kahle

Theorie und Geschichte der Wissenschaften, Universität Tübingen, Germany, and CMA, FCT, Universidade Nova de Lisboa, Caparica, Portugal e-mail: kahle@mat.uc.pt

Isabel Oitavem

CMA and DM, FCT, Universidade Nova de Lisboa, Portugal, e-mail: oitavem@fct.unl.pt

${ }^{1}$ For a more detailed historical and philosophical discussion of Gentzen's result, see Kahle 2015. 
Gentzen was well aware that this could be at best a first step towards a consistency proof for Analysis. In 1938, he wrote: ${ }^{2}$

Indeed, it seems not entirely unreasonable to me to suppose that contradictions might possibly be concealed even in classical analysis.

... the most important [consistency] proof of all in practice, that for analysis, is still outstanding. (Gentzen 1969, 235-236)

It is known that Gentzen was thinking of such a proof up to his premature death in a prison in Prague in $1945 ;^{3}$ but it is clear that he didn't reach any satisfactory solution. And modern elaborations of Gentzen-style proof theory suggest that it is unlikely that we are going to find an easy solution to the problem of determining the proof-theoretic ordinal of Analysis, for instance, in the form of second-order arithmetic as defined in Hilbert and Bernays 1939, Suppl. IV. Even today we reach "only" subsystems of Analysis, like $\Pi_{2}^{1}$ comprehension. 4

When Lorenzen attended a talk of Gerhard Gentzen in 1937 or 1938, he observed that "the problem of freedom from contradiction of the classical calculus of logic" was closely related to lattice theory, the research area of his Ph.D. thesis. ${ }^{5}$ As a result of this observation, he worked out a consistency proof for ramified type theory without reducibility, which was published in 1951 (Lorenzen 1951a) but can be traced back to the 1940s. Lorenzen also put his algebraic perspective on record: "logical calculuses are semilattices or lattices" (Lorenzen $2017, \S 4) .{ }^{6}$ The result was appreciated at the time by Paul Bernays, but today it is widely perceived as an isolated accomplishment rather than a conceptional breakthrough, let alone a philosophical solution to the consistency problem in Mathematics.

${ }^{2}$ In 1934, Bernays already highlighted the importance of a consistency proof for Analysis, with an additional twist concerning the role of intuitionistic methods:

The question which now arises is whether the strengthening of the method of proof theory obtained by admitting the abstract arguments of intuitionism would put us into a position to prove the consistency of analysis. The answer would be very important and even decisive for proof theory, and even, it seems to me, for the role which is to be attributed to intuitionistic methods. (Bernays 1964, 286; translated from the French original published in 1935)

3 Szabo (Gentzen 1969, viii) relates the memories of a prison friend of Gentzen: "He once confided in me that he was really quite contented since now he had at last time to think about a consistency proof for analysis. He was in fact fully convinced that he would succeed in carrying out such a proof."

4 See, for instance, Rathjen 2005a; 2005b. Szabo (in Gentzen 1969, 12-16) gives a short review of consistency results going beyond Arithmetic. He covers Fitch, Takeuti, and Ackermann, but also Lorenzen and Schütte.

5 See Coquand and Neuwirth 2020, $\S 1$. The contribution of Lorenzen to lattice theory and its legacy in modern developments is discussed in the contributions by Coquand, Lombardi, and Neuwirth, Neuwirth, and Schuster and Wessel in this volume.

${ }^{6}$ German orginal: "die logischen Kalküle [sind] Halbverbände oder Verbände" (Lorenzen 1951a, 89). 
Proof theory, as a specialized discipline within mathematical logic, did not take up Lorenzen's result,7 but rather developed other techniques, notably Ordinal Analysis as a tool to measure the strength of mathematical theories. In this paper, we will place the result of Lorenzen's 1951 Journal of Symbolic Logic article (1951a) in its historical context and try to explain why its legacy is ambivalent: while it doesn't occupy a distinguished status in the context of Hilbert's Programme, it provided the most useful tool in post-Gentzen proof theory: the $\omega$-rule.

In his book Beweistheorie (1960), Schütte credits Lorenzen (as already in his 1951) with the introduction of the $\omega$-rule in proof theory and devotes a good part of the final discussion of possible foundations of Analysis (Schütte 1960, §36) to Lorenzen's work (referring, in fact, not only to Lorenzen 1951a, but also touching on Lorenzen 1951b). Credit and discussion, however, vanished in the entirely rewritten English edition Proof Theory of 1977 (Schütte 1977). In Sundholm's Ph.D. thesis on the $\omega$-rule of 1983, history appears to be reverted when Lorenzen is mentioned only after Schütte's paper (1951) with the brief recognition: "The same idea is found in Lorenzen (1951), but there the ordinals are lacking" (Sundholm 1983, 1:3).

It is thus overdue to assign to Lorenzen his appropriate place in the history of the $\omega$-rule as its inventor in proof theory.

\section{Lorenzen's consistency proof for ramified type theory (without reducibility)}

In 1951, Lorenzen published his paper "Algebraische und logistische Untersuchungen über freie Verbände" (Lorenzen 1951a). It was recently translated by Neuwirth (Lorenzen 2017). Coquand and Neuwirth $(2017,1)$ provide a neat description of Lorenzen's achievement:

The "Investigations" are best known for providing a constructive proof of consistency for ramified type theory without axiom of reducibility. They do so by showing that it is a part of a trivially consistent "inductive calculus" that describes our knowledge of arithmetic without detour. The proof resorts only to the inductive definition of formulas and theorems.

And in a footnote:

More precisely, Lorenzen proves the admissibility of cut by double induction, on the cut formula and on the complexity of the derivations, without using any ordinal assignment, contrary to the presentation of cut elimination in most standard texts on proof theory.

Here, we will not discuss the mathematical details of Lorenzen's proof, but refer to the article itself (Lorenzen 1951a; 2017) and the discussion of it

7 Only recently, Coquand and Neuwirth $(2017 ; 2020)$ have revived the mathematical interest in Lorenzen's result and methods; see also the contribution by Coquand in this volume. 
by Coquand and Neuwirth (2017; 2020). We would only like to highlight two aspects which are important for a historical and philosophical evaluation of Lorenzen's work.

The first one is that Lorenzen deals "only" with Analysis (in the formal framework of ramified type theory) without reducibility. Just as his result has to be considered a breakthrough in Hilbertian proof theory, so it is limited: without reducibility one does not reach full Analysis in the form of Hilbert and Bernays's second-order arithmetic. Of course Lorenzen was aware of this, and gave a clear caveat: "As this axiom [of reducibility] is not comprised, our calculus does not contain classical analysis, ..." (Lorenzen $2017,2){ }^{8}$ The problem is not that Lorenzen considered only a rather weak form of Analysis, but that his method did not open the way to analyzing stronger systems. When Lorenzen himself turned more to foundational questions of logic (see $\S 4$ below), his approach was superseded by Schütte's proof-theoretic investigations.

The second observation, however, is a much more positive one: Lorenzen was the first to consider a proper $\omega$-rule in proof theory. The history of the $\omega$-rule is rather intricate. Within proof theory, it is often attributed to Hilbert, who-apparently-suggested such a rule in 1930, when his programme was about to fail due to Gödel's Theorems (although it is unclear whether by this time Hilbert was already informed about Gödel's results). A close inspection of Hilbert's wording in his 1931a, 1931b, however, shows that he did not propose an $\omega$-rule in a formal calculus. ${ }^{9} \mathrm{He}$ discussed a "meta-rule" which would allow to introduce a new axiom ("Anfangsformel" in German) $\forall n . A(n)$ when one has proofs for all instances with fixed natural numbers $A(0), A(1), \ldots$ (in addition, $A$ is restricted to be quantifier-free).

For the proper infinitary rule, Feferman $(1986,213)$ writes: "Apparently, the first to consider such a frankly infinitary rule was Tarski in a lecture he gave in 1927, although his first publication in which this was stated is Tarski 1933." He also refers to Carnap, who formulated such a rule in 1934 and 1935, such that Rosser, in 1937, 129, used the designation "Carnap's rule". As close as Hilbert might have been to the $\omega$-rule as we know it today, there is a bold conceptional step necessary to incorporate such a rule on the object level, to consider semi-formal systems, as we call them today (Schütte 1960; 1977). For proof-theoretic investigations, it was, in some sense, not even Lorenzen who took such a step. ${ }^{10}$ As far as we can see, he never

${ }^{8}$ German original: “Da dieses Axiom nicht miteinbezogen ist, enthält unser Kalkül nicht die klassische Analysis" (Lorenzen 1951a, 82).

9 In fact, in 1926, he had mocked infinitary proofs:

... some stress the stipulation, as a kind of restrictive condition, that, if mathematics is to be rigorous, only a finite number of inferences is admissible in a proof-as if anyone had ever succeeded in carrying out an infinite number of them! (Hilbert 1967, 370; translation of Hilbert 1926, 162)

${ }^{10}$ In our understanding, this achievement is due to Schütte; see below. 
referred to Hilbert's final papers, and may not even have read them. On the contrary, he had a completely independent motivation, which came from his expertise in lattice theory. ${ }^{11}$ Looking at derivations with infinitely many premises from an algebraic perspective, the conclusion may appear as the infimum of an infinite set. Algebra, of course, has no foundational problems in dealing with infinite objects, and an infimum is an absolutely natural object in this context. Thus, Lorenzen just realized that-existingalgebraic structures can be considered as (infinite) proof objects. This is a quite different route from first extending a calculus by an infinite rule and then giving it an algebraic interpretation. In addition, Lorenzen's motivation is entirely mathematical in nature-an aspect which would probably have delighted Hilbert, as it was his declared objective to "transfer the entire set of issues [of the foundational questions in mathematics] into the domain of pure mathematics." 12

In the following, we take a closer look at the historical context in which Lorenzen's paper developed (it had, in fact, a rather long history).

\section{Gentzen, Bernays, Schütte}

According to Lorenzen's own testimony, he attended a talk by Gentzen in 1937 or 1938, realizing that his "algebraic works ... were concerned with a problem that had formally the same structure as the problem of freedom from contradiction of the classical calculus of logic."13 In 1944, at the latest, he had a draft for a consistency proof for elementary number theory along these lines, "Ein halbordnungstheoretischer Widerspruchsfreiheitsbeweis" (Lorenz 2020; see also Coquand and Neuwirth 2020). ${ }^{14}$ By December 1945, he had extended the result to classical logic with ramified type theory (Coquand and Neuwirth 2017, §3); Heinrich Scholz submitted the corresponding

\footnotetext{
${ }^{11}$ For his algebraic education under Hasse and Köthe, see Coquand and Neuwirth 2020, $\S 1$.

12 The full German passage from Hilbert's talk at the International Congress of Mathematicians in Rome in 1928 reads as follows (Hilbert 1928, 3):
}

Mit dieser Neubegründung der Mathematik, die man füglich als eine Beweistheorie bezeichnen kann, glaube ich die Grundlagenfragen in der Mathematik als solche endgültig aus der Welt zu schaffen, indem ich jede mathematische Aussage zu einer konkret aufweisbaren und streng ableitbaren Formel mache und dadurch den ganzen Fragenkomplex in die Domäne der reinen Mathematik versetze.

13 See Coquand and Neuwirth 2020, $\S 1$ and footnote 5, with reference to a letter by Lorenzen to Carl Friedrich Gethmann.

${ }^{14}$ Coquand and Neuwirth $(2020, \S 2)$ provide some evidence that the paper was sent at that time to Wilhelm Ackermann, Gerhard Gentzen, Hans Hermes, and Heinrich Scholz. 
manuscript to Paul Bernays. ${ }^{15}$ In 1947, Lorenzen sent a new version to Bernays, which, according to Coquand and Neuwirth $(2017, \S 4)$, "tries to make a synthesis of 'Ein halbordnungstheoretischer Widerspruchsfreiheitsbeweis' and 'Die Widerspruchsfreiheit der klassischen Logik mit verzweigter Typentheorie', but is rather a juxtaposition of the two: the seams remain apparent." Its first part contains an exposition of the lattice-theoretic background. After some discussion with Bernays (see below and Coquand and Neuwirth 2017), the paper was submitted to the Journal of Symbolic Logic on March 17, 1950, and published in 1951 (Lorenzen 1951a).

Gerhard Gentzen still responded to Lorenzen on the 1944 version, with a rather discouraging remark: "The freedom from contradiction of number theory cannot be proven so simply."16 Lorenzen would hardly have been excited about Gentzen's reaction, and nearly 50 years later he commented on it in a letter to Menzler-Trott: "Contrary to Gentzen's opinion, consistency proofs do allow themselves to be 'so simply' proven."17

The reaction of Bernays-who could have seen only later versions already dealing with Analysis—was, however, quite enthusiastic: ${ }^{18}$

It seems to me that your argumentation accomplishes in effect the desired and that thereby at the same time also a new, methodically more transparent proof of consistency for the number-theoretic formalism, as well as for Gentzen's subformula theorem is provided.

In particular, he was able to clarify Lorenzen's metamathematical base by indicating that he starts from a constructive rather than a finite standpoint. In a letter from February 21, 1947, Lorenzen wrote to Bernays:

I beg once again to ask you for your advice-namely, it is not clear to me whether I rightly call the logic used here "finite" logic.

Bernays replied on April 3, 1947:

When it comes to the methodical standpoint and to the terminology to be used in relation, then it seems advisable to me to keep with the mode chosen by Mr Gentzen, that one speaks of "finite" reflections only in the narrower sense, i.e. relating to considerations that may be formalised in the framework of recursive

15 The letter is contained in the Bernays Nachlass at the ETH Zurich, HS. 975:4111; a copy of the manuscript can be found in the Nachlass of Köthe at the Niedersächsische Staatsund Universitätsbibliothek Göttingen, Cod. Ms. G. Köthe M 10 (Coquand and Neuwirth 2020, footnote 17).

${ }^{16}$ Letter by Gentzen to Lorenzen from September 12, 1944. The full text of the letter in German is given in Menzler-Trott 2001, 259-260, and reproduced photographically (Menzler-Trott 2001, 372).

17 German original: "Wf-beweise lassen sich — gegen die Meinung Gentzens - doch ,so einfach' beweisen" (Menzler-Trott 2001, 260, footnote 31); for the English translation, see Menzler-Trott 2007. We note in passing that by that time, Lorenzen thought he had sent to Gentzen already a version of his paper containing the consistency proof for Analysis.

18 The translation of the correspondence between Lorenzen and Bernays is taken from Coquand and Neuwirth 2017, where one also finds the original German texts and the references to the sources. 
number theory (possibly with extension of the domain of functions to arbitrary computable functions), that one uses in contrast the expression "constructive" for the appropriate extension of the standpoint of the intuitive self-evidence.

Lorenzen happily accepted this terminological shift (which, of course, corresponded to a substantial conceptional alteration, compared to Hilbert's original conception):

Your proposal to call the means of proof not "finite" but "constructive" acted on me as a sort of redemption. I was sticking so far to the word finite only to emphasise that these are Hilbertian ideas that I am trying to pursue.

With Paul Bernays, Lorenzen had of course the highest authority in Hilbertian proof theory of that time on his side. However, Lorenzen was not really engaged in the proof-theoretic school of Hilbert, which had remained in Germany and was led by Arnold Schmidt. ${ }^{19}$

Through Bernays or Schmidt, Kurt Schütte would have received a copy of Lorenzen's article. ${ }^{20}$ In 1948/49, Schütte developed his own proof-theoretic methods, which make substantial use of the $\omega$-rule, taken from Lorenzenin 1950, he wrote in his letter to Lorenzen: "This insight gained by you which appears to me exceptionally important for fundamental research, I have now taken up." 21

When describing his results in a letter to Paul Bernays of August 26, 1949, he refers to Lorenzen in two respects. ${ }^{22}$ First, he credits Lorenzen with the invention of the $\omega$-rule:

Herein the following are utilized: ... 2. the possibility, discovered by Lorenzen, of preserving Gentzen's Hauptsatz also under inclusion of formal induction, namely by means of inferences with an infinite number of premises, ...

In a following remark, however, he makes the link to Hilbert's latest publication:

19 In a letter to Bernays from April 30, 1950 (Hs. 975:3926 in the Bernays Nachlass at the ETH Zurich), Schmidt expresses a quite negative impression of Lorenzen: "Mr. Lorenzen sent me a draft on decision in positive logic.... His proofs are very sophisticated, but also 'in the details' often downright incorrect and only reparable with new methodological changes (but that doesn't interest him, if you call his attention to it, and doesn't shock him at all)." ("Herr Lorenzen sandte mir einen Entwurf zur Entscheidung in der positiven Logik. ... Seine Beweise sind sehr elegant, allerdings auch ,im kleinen' oft regelrecht unrichtig und nur mit neuen methodischen Wendungen reparabel (was ihn aber, wenn man ihn darauf aufmerksam macht, nicht interessiert und nicht im geringsten erschüttert).") In view of the later developments in Hilbert-style proof theory, which heavily relies on technical accuracy, Lorenzen's attitude (as observed by Schmidt) is, of course, devastating. ${ }^{20}$ See the opening of the letter by Schütte to Lorenzen from May 1, 1950 (PL 1-1-45 in the Lorenzen Nachlass kept at the University of Konstanz). The letter suggests that Schütte and Lorenzen did not know each other from before. Lorenzen studied in Göttingen from 1936 to 1939, when Schütte had already left the University.

${ }^{21}$ See Coquand and Neuwirth 2017, §6, in particular, footnote 35, for a longer passage of the letter.

22 The letter is reprinted and discussed in its historical context in Kahle 2020. 
2. with the inference of "infinite induction"

$$
\frac{\mathfrak{A}(\mathfrak{z}) \vee \mathfrak{N} \quad \text { for all numerals } \mathfrak{z}}{(x) \mathfrak{A}(x) \vee \mathfrak{N}}
$$

as it was accordingly already recommended by Hilbert.

This link is misleading, as Hilbert draws only on a metamathematical rule to introduce new universal axioms. Schütte clearly uses an infinite rule on the object level. By conflating this with Hilbert's proposal he implemented a historical misunderstanding; on the other hand, he fully released the $\omega$-rule from the mathematical motivation present in Lorenzen's work and turned it, as such, into a new and independent tool in proof theory. Schütte did not only give thanks to Lorenzen in the letter mentioned above, but also credited him as the first one using a rule with infinitely many premisses for a consistency proof in his publication Schütte 1951 as well as in his monograph Beweistheorie (Schütte 1960, 168).

Secondly, Schütte was very much concerned to convince Bernays that his approach was sufficiently different from Lorenzen's to deserve publication:

This proof idea is entirely in keeping with the one applied by Lorenzen, but the implementation is a slightly different one. I think, that my investigations are not redundant when put next to those of Lorenzen because the necessary metamathematical means of proof and the connections with the deducibility of the formalized transfinite induction are revealed in this way.

and:

The results should relate to those of Lorenzen in that the sentence induction [Satzinduktion] employed by Lorenzen is of the same character of a transfinite induction over the second number class as the transfinite induction ranging over the orders of derivations. If one allows arbitrary inferences with infinitely many premises, as is the case with Lorenzen, the corresponding transfinite induction will reach arbitrarily high levels of the second number class.

Schütte's work shifted away the analysis from the mathematical (latticetheoretic) background, highlighted by Lorenzen. Conceptionally, he was bringing back the ordinals of Gentzen (which are completely absent in Lorenzen's work). In the long run, this was a decisive advantage for Schütte's approach, as it paved the way for the analysis of other mathematical theories. ${ }^{23}$

23 One should add here that modern proof theory is of course not just interested in consistency proofs, but rather in additional information. In this respect, Macintyre (2005, 2426) writes:

Much nonsense has been pronounced about Gentzen's work, even by extremely distinguished people. Consistency is not really the main issue at all. He did reveal fine structure in the unprovability of consistency of PA, as a consequence of much deeper general methodology. 


\section{Digression: Lorenzen's 1951 Zeitschrift paper}

Although going back to work from the 1940s, Lorenzen's paper "Algebraische und logistische Untersuchungen über freie Verbände" appeared in print only in 1951 in the Journal of Symbolic Logic. By that time, Lorenzen's interest had already shifted. ${ }^{24}$ By coincidence, it was also in 1951 that another paper of Lorenzen appeared, in the Mathematische Zeitschrift, with thequite misleading-title "Die Widerspruchsfreiheit der klassischen Analysis" ("The consistency of classical analysis"; Lorenzen 1951b). One has to remember that in his JSL paper Lorenzen clearly stated that, due to the missing reducibility axiom, his consistency proof does not extend to classical Analysis. ${ }^{25}$ In the Zeitschrift paper, which has no relation to the JSL paper, he is not even attempting to provide a consistency proof. In contrast, he provides a constructive (re-)definition of Analysis, based on a "construction of the real numbers for which all fundamental theorems of classical analysis are valid" (Lorenzen 1951b, 1). These real numbers are constructed in stages, and to obtain them, one has to take the union of the finite stages (only!). Thus, it is necessary to adapt the classical theorems to this framework! ${ }^{26}$ In consequence, one can hardly call the system under consideration "classical analysis". And, as Kurt Schütte stated this in his review of Lorenzen 1951b in the Journal of Symbolic Logic (Schütte 1953, 261): "the author carries out a construction of real numbers, which does not give the usual classical analysis, but a system in which all corresponding fundamental theorems are valid."

The problem of the 1951 paper is not only the misleading title; it also marks a significant turn of Lorenzen away from Hilbert's philosophical basis. In a tactical move, Hilbert had proposed finite mathematics as foundation to secure ordinary mathematics; it was somehow the goal to beat Brouwer at his own game. Gödel's Theorems show that this cannot be carried out. For Hilbert, however, this is not an argument to dismiss parts of Mathematics; on the contrary, the foundational base has to be adapted. This led, first, to the change from finite to constructive mathematics. ${ }^{27}$ Today, we have to admit that even constructive mathematics will not be sufficient. ${ }^{28}$ From a

\footnotetext{
24 "In fact, from 1947 on, Lorenzen is already mostly occupied by his project of layers of language which will lead to his operative logic" (Coquand and Neuwirth 2017, end of $§ 5$ ). Operative logic and mathematics preceded Lorenzen's introduction of dialogical logic and deserves its own recognition; see the contribution by Heinzmann in this volume.

${ }^{25}$ See footnote 8 above.

${ }^{26}$ Such (necessary) reinterpretations of classical theorems are still today in the focus of research in constructive analysis.

27 See, in particular, the remark of Bernays to Lorenzen in his letter of April 3, 1947 above. ${ }^{28}$ See Martin-Löf's comment concerning the alleged "second failure of Hilbert's program":
}

This is what I propose to call the second failure of the Hilbert program. The first failure of the Hilbert program was the one which was discovered by Gödel and of which we are now all aware, but that gave rise to the revised, or modified, Hilbert 
Hilbertian perspective, this only necessitates another liberalization of the foundational standpoint. Lorenzen, however, turned into a "constructive fundamentalist", as we may say. In Lorenzen 1968, for instance, he made clear that classical mathematics is "justified", at best, by a constructive interpretation or a constructive consistency proof. ${ }^{29}$

Lorenzen still worked out constructive approaches to Analysis (see his textbook on Differential and Integral, Lorenzen 1965), but eventually turned completely to logic and philosophy. ${ }^{30}$ Doing so, he contributed substantially to the philosophical discussion of the foundations of logic, but the link to proof theory in the spirit of Hilbert was lost.

\section{Conclusion}

Lorenzen's consistency proof for ramified type theory without reducibility has to be considered as a landmark in proof theory. It is distinguished by the introduction of the $\omega$-rule and the underlying mathematical methodology stemming from lattice theory.

Lorenzen was commissioned to write the chapter on Foundations of Mathematics for the FIAT report ${ }^{31}$ on Pure Mathematics (Lorenzen 1948). From it, one can obtain an impression of how Lorenzen himself judged the importance of his work, when he devoted nearly 4 of the 12 pages to his own unpublished manuscripts, more than half of them in the section on "Finite Logistics", 32 which otherwise covers only Gentzen and Ackermann.

When afterwards Lorenzen turned, first, to constructive reformulations of classical mathematics and, later, to philosophical foundations of logic, he

program, whose characteristic is that we no longer allow merely combinatorial methods in the consistency proof but arbitrarily strong constructive methods. But even this revised, or modified, Hilbert program has come to an end in the nineties, or has failed in the nineties, so it is the second failure of the original Hilbert program, which I cannot interpret in any other way than that we have to give up the dream of being able to establish the consistency of classical mathematics by constructive means. (Martin-Löf 2008, 254)

Here one can speak of a "failure" only if one takes the constructive point of view as the last resort for foundations.

29 Occasionally, however, Lorenzen was rather liberal in his discussions: in the preface to his book on Metamathematics, he comes to the-correct-conclusion: "There exists no contradiction between constructive and classical set theory" (Lorenzen 1962, 9).

$3^{0}$ This turn is described in part in the recollections of his student Kuno Lorenz in this volume.

${ }^{11}$ After World War II, the US Army was collecting in the FIAT ("Field Information Agency, Technical") reports information about the development of science in Germany during the years 1939-1946.

32 The section heading suggests that he finished this report before receiving Bernays's suggestion to switch from "finite" to "constructive". 
lost the "mathematical momentum" of his consistency proof. As a result, mathematical proof theory changed course. The $\omega$-rule turned into a basic tool in ordinal analysis, under the guidance of Kurt Schütte.

But it is worth noting that the mathematical inspiration for Lorenzen's consistency proof fits squarely within Hilbert's philosophical vision. To determine its full rationale within Hilbertian proof theory and its potential for the investigation of stronger mathematical theories is, however, still a desideratum.

\section{References}

Bernays, Paul. 1964. "On platonism in mathematics." In Philosophy of mathematics, edited by Paul Benacerraf and Hilary Putnam, pages 274286. Prentice-Hall. Lecture delivered June 18, 1934, in the cycle of Conferences internationales des sciences mathematiques organized by the University of Geneva, in the series on Mathematical Logic. Translated from the French by C. D. Parsons from L'enseignement mathematique, 1st ser., vol. 34 (1935), pp. 52-69.

Carnap, Rudolf. 1935. "Ein Gültigkeitskriterium für die Sätze der klassischen Mathematik." Monatshefte für Mathematik und Physik 42:163-190.

Coquand, Thierry. 2020. "Lorenzen and constructive mathematics." In this volume, pages $45-59$.

Coquand, Thierry, Henri Lombardi, and Stefan Neuwirth. 2020. "Regular entailment relations." In this volume, pages 101-112.

Coquand, Thierry, and Stefan Neuwirth. 2017. An introduction to Lorenzen's "Algebraic and logistic investigations on free lattices" (1951). arXiv: 1711.06139vi [math.LO].

- 2020. "Lorenzen's proof of consistency for elementary number theory." History and Philosophy of Science. https://doi.org/10.1080/ 01445340.2020 .1752034$.

Feferman, Solomon. 1986. "Introductory note to [Gödel 1931c]." In Kurt Gödel: Collected works, I: Publications 1929-1936, edited by S. Feferman et al., pages 208-213. Oxford University Press.

Gentzen, Gerhard. 1936. "Die Widerspruchsfreiheit der reinen Zahlentheorie." Mathematische Annalen 112:493-565.

- 1969. Collected works. Edited by M. E. Szabo. North-Holland.

Gödel, Kurt. 1931. “Über formal unentscheidbare Sätze der Principia Mathematica und verwandter Systeme I." Monatshefte für Mathematik und Physik 38:173-198.

Heinzmann, Gerhard. 2020. “Operation and predicativity: Lorenzen's approach to arithmetic." In this volume, pages 9-20.

Hilbert, David. 1926. "Über das Unendliche." Mathematische Annalen 95:161-190. 
. 1928. "Probleme der Grundlegung der Mathematik." In Atti del Congresso Internazionale dei Matematici (Bologna, 3-19 settembre 1928). Nicola Zanichelli. Offprint.

—. 1931a. "Beweis des Tertium non datur." Nachrichten von der Gesellschaft der Wissenschaften zu Göttingen, Mathematisch-Physikalische Klasse, pages 120-125. Talk given on July 17, 1931 in Göttingen.

—. 1931b. "Die Grundlegung der elementaren Zahlenlehre." Mathematische Annalen 104 (1): 485-494. Talk given in December 1930 in Hamburg.

- 1967. "On the infinite." In From Frege to Gödel: A source book in mathematical logic, 1879-1931, edited by Jean van Heijenoort, pages 367-392. Harvard University Press. English translation of Hilbert 1926. Hilbert, David, and Paul Bernays. 1939. Grundlagen der Mathematik II. Die Grundlehren der mathematischen Wissenschaften in Einzeldarstellungen, 50. Springer. Second edition 1970.

Kahle, Reinhard. 2015. "Gentzen's theorem in context." In Gentzen's centenary: The quest for consistency, edited by Reinhard Kahle and Michael Rathjen, pages 3-24. Springer.

- 2020. "'Sehr geehrter Herr Professor!' Proof theory in 1949 in a letter from Schütte to Bernays." In The legacy of Kurt Schütte, edited by Reinhard Kahle and Michael Rathjen, pages 3-19. Springer.

Lorenz, Kuno. 2020. "Paul Lorenzens Weg von der Mathematik zur Philosophie: Persönliche Erinnerungen." In this volume, pages 1-8.

Lorenzen, Paul. 1948. "Grundlagen der Mathematik." In Reine Mathematik, Naturforschung und Medizin in Deutschland 1939-1946 (für Deutschland bestimmte Ausgabe der FIAT Review of German Science), Band 1, Teil 1, edited by W. Süss, pages 11-22. Dietrich'sche Verlagsbuchhandlung.

—. 1951a. "Algebraische und logistische Untersuchungen über freie Verbände." Journal of Symbolic Logic 16:81-106.

- 1951b. "Die Widerspruchsfreiheit der klassischen Analysis." Mathematische Zeitschrift 54:1-24.

- 1962. Metamathematik. Volume 25 of BI Hochschultaschenbücher. Bibliographisches Institut.

- 1965. Differential und Integral: Eine konstruktive Einführung in die klassische Analysis. Akademische Verlagsgesellschaft.

- 1968. "Constructive mathematics as a philosophical problem." Compositio Mathematica 20:133-142. Also published (with identical pagination) in Logic and foundations of mathematics, edited by D. van Dalen, J. G. Dijkman, S. C. Kleene, and A. S. Troelstra, Wolters-Noordhoff Publishing.

- 2017. "Algebraic and logistic investigations on free lattices." arXiv: 1710.08138. Translation by Stefan Neuwirth of Lorenzen 1951a.

- (1944) 2020. "Ein halbordnungstheoretischer Widerspruchsfreiheitsbeweis." History and Philosophy of Logic. From the Oskar Becker Nachlass, Philosophical Archive of the University of Konstanz, file 
OB 5-3b-5, edited and translated as "A proof of freedom from contradiction within the theory of partial order" by Stefan Neuwirth. https://doi.org/10.1080/01445340.2020.1752040

Macintyre, Angus. 2005. "The mathematical significance of proof theory." Philosophical Transactions of the Royal Society A 363:2419-2435.

Martin-Löf, Per. 2008. “The Hilbert-Brouwer controversy resolved?" In One hundred years of intuitionism (1907-2007): The Cerisy conference, edited by Mark van Atten, Pascal Boldini, Michel Bourdeau, and Gerhard Heinzmann, pages 243-256. Birkhäuser.

Menzler-Trott, Eckhart. 2001. Gentzens Problem. Birkhäuser.

- 2007. Logic's lost genius: The life of Gerhard Gentzen. Volume 33 of History of mathematics. American Mathematical Society.

Neuwirth, Stefan. 2020. "Lorenzen's reshaping of Krull's Fundamentalsatz for integral domains (1938-1953)." In this volume, pages 141-180.

Rathjen, Michael. 2005a. "An ordinal analysis of parameter free $\Pi_{2}^{1}$ comprehension." Archive for Mathematical Logic 44 (3): 263-362.

- 2005b. "An ordinal analysis of stability." Archive for Mathematical Logic 44 (1): 1-62.

Rosser, Barkley. 1937. "Gödel theorems for non-constructive logics." Journal of Symbolic Logic 2 (3): 129-137.

Schuster, Peter, and Daniel Wessel. 2020. "Syntax for semantics: Krull's maximal ideal theorem." In this volume, pages 75-100.

Schütte, Kurt. 1951. "Beweistheoretische Erfassung der unendlichen Induktion in der Zahlentheorie." Mathematische Annalen 122:369-389.

- 1953. Review of Paul Lorenzen, Die Widerspruchsfreiheit der Klassischen Analysis (Lorenzen 1951a). Journal of Symbolic Logic 18 (3): 261-262.

- 1960. Beweistheorie. Volume 103 of Grundlehren der Mathematischen Wissenschaften. Berlin: Springer.

. 1977. Proof theory. Springer.

Sundholm, Göran. 1983. "Proof theory: A survey of the omega-rule." Ph.D. dissertation, Magdalen College, University of Oxford.

Tarski, Alfred. 1933. "Einige Betrachtungen über die Begriffe der $\omega$-Widerspruchsfreiheit und der $\omega$-Vollständigkeit." Monatshefte für Mathematik und Physik 40:97-112. 
Open Access This chapter is licensed under the terms of the Creative Commons Attribution 4.0 International License (http://creativecommons.org/licenses/by/4.0/), which permits use, sharing, adaptation, distribution and reproduction in any medium or format, as long as you give appropriate credit to the original author(s) and the source, provide a link to the Creative Commons license and indicate if changes were made.

The images or other third party material in this chapter are included in the chapter's Creative Commons license, unless indicated otherwise in a credit line to the material. If material is not included in the chapter's Creative Commons license and your intended use is not permitted by statutory regulation or exceeds the permitted use, you will need to obtain permission directly from the copyright holder.

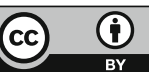

